\title{
Transcranial Magnetic Stimulation and Aphasia Rehabilitation
}

\author{
Margaret A. Naeser, Ph.D. ${ }^{1}$, Paula I Martin, B.S. ${ }^{1}$, Michael Ho, Ph.D. ${ }^{1}$, Ethan Treglia, M.S., \\ CCCSLP ${ }^{1}$, Elina Kaplan, B.S. ${ }^{1}$, Shahid Bhashir, Ph.D. ${ }^{2}$, and Alvaro Pascual-Leone, M.D., \\ Ph.D. ${ }^{2,3}$ \\ ${ }^{1}$ Veterans Affairs Boston Healthcare System and the Harold Goodglass Boston University \\ Aphasia Research Center, Department of Neurology, Boston University School of Medicine \\ ${ }^{2}$ Berenson-Allen Center for Noninvasive Brain Stimulation, Department of Neurology, Harvard \\ Medical School and Beth Israel Deaconess Medical Center \\ ${ }^{3}$ Institut Guttman de Neurorehabilitació, Institut Universitari de la Universitat Autonoma de \\ Barcelona, Badalona, Spain
}

\begin{abstract}
Repetitive transcranial magnetic stimulation (rTMS) has been reported to improve naming in chronic stroke patients with nonfluent aphasia since 2005. In Part 1, we review the rationale for applying slow, $1 \mathrm{~Hz}$, rTMS to the undamaged right hemisphere in chronic nonfluent aphasia patients following a left hemisphere stroke; and present a TMS protocol used with these patients that is associated with long-term, improved naming post- TMS. In Part, 2 we present results from a case study with chronic nonfluent aphasia where TMS treatments were followed immediately by speech therapy (constraint-induced language therapy). In Part 3, some possible mechanisms associated with improvement following a series of TMS treatments in stroke patients with aphasia are discussed.
\end{abstract}

\section{Keywords}

Aphasia; TMS; stroke rehabilitation

\section{Transcranial Magnetic Stimulation}

Repetitive transcranial magnetic brain stimulation (rTMS) has been studied worldwide since $1985^{1}$ as a potential treatment for some disorders associated with stroke, including paralysis or hemispatial visual neglect; as well as to treat other disorders such as depression and epilepsy. ${ }^{2}$ This paper presents an overview of rTMS where this new technology is explained in relationship to treatment of aphasia. In Part 1, we present the rationale for using rTMS on the right hemisphere (RH) in chronic nonfluent aphasia patients with left hemisphere (LH)

\footnotetext{
Address correspondence to: Margaret Naeser, Ph.D., Aphasia Research Center (12-A), V.A. Boston Healthcare System, 150 So. Huntington Ave., Boston, MA 02130 U.S.A., Phone: 857-364-4030 FAX: 617 739-8926 mnaeser@bu.edu.

Device Status: There are various manufacturers of TMS devices. The device by Neuronetics is FDA approved for treatment of medication refractory depression, but lacks the flexibility and precision needed for the present application. In the present study we use the device manufactured by Magstim, Magstim Super Rapid, equipped with an 8-shaped stimulation coil. Both the device and the coil have been cleared through the Premarket Notification $(510(\mathrm{k}))$ process as being substantially equivalent to electric stimulation devices. The transcranial application to promote aphasia recovery is thus an off-label application of this approved device. Given the extensive safety data and the strict adherence of our study protocol to current safety guidelines and recommendations endorsed by the International Federation for Clinical Neurophysiology, the Committee for Clinical Investigations (CCI) at Beth Israel Deaconess Medical Center, Boston, agreed with the assessment that the application of the Magstim Super Rapid TMS device following the Naeser et al., protocol represents a 'non-significant risk' application. Thus no IDE was necessary.

The legal/regulatory status of the device(s) by others reviewed in this manuscript is/are not known, by the author(s).
} 
stroke. We also present a TMS protocol associated with long-term, improved naming postTMS treatment, and review a related functional MRI (fMRI) study. In Part 2, we briefly review a case study where TMS was combined with Constraint-Induced Language Therapy (CILT). In Part 3, we conclude with a review of possible mechanisms underlying language improvement post- TMS.

TMS is a noninvasive procedure that utilizes magnetic fields to create electric currents in discrete brain areas. ${ }^{3,4}$ TMS involves discharging a current through a coil of copper wire that is held over the subject's scalp. The current pulse flowing through the coil generates a rapidly fluctuating magnetic field that penetrates the scalp and skull unimpeded, and induces a changing electrical field in the cerebral cortex below the coil. The physiologic response appears to be caused by current flow in the cortical tissue, which leads to neuronal depolarization, exciting or inhibiting the cortex. ${ }^{5}$ The participant feels a "light tap" on the scalp, may feel a twitch of the face muscles, and hears a brief, loud click as the current passing through the coil tightens the copper wire. Participants report that this is not unpleasant. The stimulation of the brain itself is painless.

When rTMS is applied as multiple stimuli ("trains") of appropriate frequency, intensity, and duration, rTMS can lead to increases or decreases in excitability of the affected cortex that last beyond the duration of the train itself. ${ }^{6}$ Slow rTMS, where one magnetic pulse is applied every second $(1 \mathrm{~Hz})$, delivered to the motor cortex can give rise to a lasting decrease in corticospinal excitability. ${ }^{7,8}$ Conversely, fast rTMS $(5,10$, or $20 \mathrm{~Hz})$ can induce a transient increase in cortical excitability. ${ }^{9}$ The maximum output of a TMS device can reach up to 2.5 Tesla. To achieve focal brain stimulation, rTMS is often applied with a figure 8-shaped stimulation coil ( $7 \mathrm{~cm}$ in diameter), where the area of brain cortex affected is approximately one cubic centimeter, located in the center where the two wings of the figure 8 -shaped coil meet.

\section{Part 1. Rationale for TMS, and a TMS Treatment Protocol for Aphasia}

\section{1a. Rationale}

Functional imaging studies of language in patients with nonfluent aphasia frequently reveal an increased activation (possible "over-activation") in RH language homologues. ${ }^{10-14}$ It is possible that unusually high activation in the RH is related to transcallosal disinhibition leading only to partial, or incomplete recovery. Such increased RH activation would represent maladaptive plasticity, and lead to a dead-end, inefficient strategy for recovery. ${ }^{2,10,12,14,15}$

Several studies have suggested that for long-term recovery, RH recruitment may be less efficient than restoring the LH network. Patients with better recovery have been observed to have higher activation in $\mathrm{L}$ superior temporal gyrus and $\mathrm{L}$ supplementary motor area (SMA). ${ }^{16-18}$ A study using perfusion-weighted imaging in acute stroke patients has shown improved naming to be associated with reperfusion of L Brodmann area (BA) 37. ${ }^{19}$ As early as 2 weeks poststroke onset, better performance on a verbal fluency test (and better recovery) was found to be associated with activation of the left inferior frontal gyrus (IFG). ${ }^{20}$

After speech-language therapy in some chronic stroke patients, new LH activation has been associated with improved language. ${ }^{21-24}$ However, in some studies, new RH activation has also been observed following speech-language therapy ${ }^{25-28}$ Right hemisphere participation in the acute recovery stage of LH stroke may be followed later, by LH activation corresponding to further recovery; the RH may play a larger role in supporting recovery when there is greater damage to LH language areas. ${ }^{29}$ It thus remains unclear whether 
recovery in aphasia is mediated primarily from LH undamaged language or perilesional regions, or from RH language homologues, or from both. It is possible that different mechanisms may be engaged by different individuals. Regardless, there seems to be potential for brain re-organization and improved language, even in chronic, post-stroke aphasia. ${ }^{15,30-32}$

Naeser and colleagues ${ }^{33}$ have hypothesized that suppression of a cortical region of interest (ROI) in the RH, with $1 \mathrm{~Hz}$ rTMS could result in a decrease of "over-activation" in that ROI, and in some patients, lead to an overall modulation of the bilateral neural network for naming. This may include re-activation of some areas within the damaged LH, and ultimately a functional language improvement. This notion is consistent with the phenomenon of 'Paradoxical Functional Facilitation' or $\mathrm{PFF}^{34}$ that suggests direct or indirect neural "damage" or disruption of a specific area in the central nervous system, may result in facilitation of behavior across functional neural networks. ${ }^{35}$

\section{1b. TMS Treatment Protocol with Nonfluent Aphasia Patients}

The studies by Naeser and colleagues, $33,36,37$ have included chronic aphasia patients who are at least 6 months post- single, unilateral LH stroke. They were R-handed, native English speakers, ranging in age from 40-73 years (allowing up to age 80). If there was a history of seizures, they were well controlled with medication, and the patient had not had a seizure for at least one year, prior to entry. Slow, $1 \mathrm{~Hz}$ rTMS, however, has been used to help treat seizures. ${ }^{38}$ Patients had nonfluent speech, with a $1-4$ word phrase length as measured with elicited propositional speech on the Cookie Theft Picture, Boston Diagnostic Aphasia Exam $^{39,40}$ (BDAE). Patients named at least 3 pictures out of 60 on the Boston Naming $\mathrm{Test}^{41}$ (BNT), but not more than 47 (to allow for potential improvement). The primary language outcome measures were the BNT and naming subtests on the BDAE.

In addition, prior to any rTMS sessions, a baseline naming ability for Snodgrass \& Vanderwart ${ }^{42}(\mathrm{~S} \& \mathrm{~V})$ pictures was established. During the baseline $\mathrm{S} \& \mathrm{~V}$ naming testing, ten, 20 -item $S \& V$ picture lists were administered. Across the ten $S \& V$ lists, the baseline, mean number of $S \& V$ pictures named correctly was calculated, as well as the baseline, mean response time $(\mathrm{RT})$.

\section{Phase 1 TMS, Locate the "best-response" RH ROI to Suppress with TMS}

During Phase 1 of TMS in the studies by Naeser and colleagues ${ }^{43}$, the "best-response" RH cortical ROI was located for each patient. This ROI was defined as that ROI, which when targeted with $1 \mathrm{~Hz}$ rTMS for 10 minutes, resulted in an immediate, significant improvement in naming, as compared to baseline naming. (This improvement in naming is only temporary, during the Phase 1 TMS protocol.) This ROI later becomes the targeted location for rTMS during Phase 2 TMS (20 minutes of rTMS, for 10 days), for that patient.

In the protocol of Naeser and colleagues ${ }^{43}$, during Phase 1 TMS, the effect of slow, $1 \mathrm{~Hz}$ rTMS for 10 min was used to suppress activity in each of at least four different RH frontal ROIs, in separate TMS sessions. A total of 600 magnetic pulses at $90 \%$ of motor threshold (MT) for the left first dorsal interosseus muscle (L FDI) was delivered to each RH ROI using the Super-Rapid High Frequency Magnetic Stimulator magstim, UK. Published guidelines for safety parameters of rTMS are based on stimulation intensities expressed as a percent of the individual's motor threshold (MT). ${ }^{44-46}$ A figure- 8 shaped rTMS coil with a 7 $\mathrm{cm}$ outside diameter on each wing was used. The RH cortical ROIs that were examined included the R primary motor cortex representation for the mouth (orbicularis oris muscle, as verified with motor evoked potentials), and at least three subregions within R Broca's area 
homologue, as described below. These subregions were labeled according to sulcal and gyral boundaries. ${ }^{36,47,48}$ See Figure 1.

The vertical ascending ramus is traditionally, the most common sulcus used to separate the pars opercularis (POp), from the pars triangularis (PTr) within Broca's area. However, in some cases, a diagonal sulcus is present. Cytoarchitectonic studies have observed, for example, that a diagonal sulcus was present in every second hemisphere, and it can either mark the border between BA 44 (likely POp) and BA 45 (likely PTr), or be located inside BA $44 .{ }^{47}$ Thus, when a diagonal sulcus is present, without cytoarchitectonics, it is not possible to know whether the diagonal sulcus is actually a border between POp and PTr, or if it is within POp. Taking this into consideration, when a diagonal sulcus is present in an aphasia patient who is participating in TMS treatment, it is important to carefully examine at least four subregions within R Broca's homologue as shown in Fig. 1.36

The exact location of the "best response" RH ROI to suppress with 1 Hz rTMS can vary somewhat, from patient to patient, and the ROI needs to be firmly established for each case. Suppression of POp often impairs naming and/or increases RT. ${ }^{43}$ The "best response" area is often reported to be located immediately anterior to the POp - e.g., R posterior PTr. ${ }^{33,36,37,49,50}$

A frameless stereotaxic system brainsight, Rogue Industries, Montreal is used in combination with the patient's 3D MPRAGE MRI scan, in order to guide the rTMS coil placement onto the targeted ROI. After each 10-min rTMS application to a specific RH ROI during Phase 1, a 20 -item S\&V set of pictures is presented for the patient to name. A "best response" RH ROI is defined as that ROI which is associated with a S\&V naming score that is at least $2 \mathrm{SD}$ above the baseline, mean number of $S \& V$ pictures named correctly.

\section{Phase 2 TMS, Two-week Treatment to Suppress the "best-response" RH ROI with TMS}

In the studies by Naeser and colleagues, $33,36,37,49$ during Phase 2 , the "best response" RH ROI from Phase 1 is targeted for 20 min of rTMS, once a day, five days a week, for two weeks. On each day of treatment, the rTMS is applied at $1 \mathrm{~Hz}$ frequency (1200 pulses) at $90 \%$ of MT (L FDI), using the same magnetic stimulator device magstim, UK as in Phase 1. The frameless stereotaxic system brainsight, Rogue Industries, Montreal is again used to guide the location of the rTMS coil on the patient's scalp. On-line monitoring allows documentation of accurate targeting of the "best response" RH ROI throughout the TMS session, and from day-to-day. Coil orientation is held constant across sessions, at approximately 45 degrees. No side effects or complications have been reported with these parameters. ${ }^{45,46}$ In order to test for possible long-term effects post- TMS, language testing is completed at 2 and at 6 months post- Phase 2 TMS.

\section{1c. Results, Language Outcome Measures Post- Phase 2 TMS}

Naeser and colleagues ${ }^{33}$ have reported at two months post- ten rTMS treatments to suppress the R PTr, significant improvement on three naming tests was observed: 1) the BNT, first 20 items ( $\mathrm{p}=.003) ; 2)$ the BDAE subtest, Animals $(\mathrm{p}=.02)$; and 3) the BDAE subtest, Tools/ Implements $(\mathrm{p}=.04)$ in four chronic nonfluent aphasia patients. At 8 months post- TMS, all three naming test scores continued to improve relative to pre- TMS testing, but only Tools/ Implements was still significant ( $\mathrm{p}=.003)$. BNT and naming Animals failed to reach significance because of one of the four patients. Improvement was also observed in number of words per longest phrase length in elicited, propositional speech (BDAE) for two of the patients at 2 months post- TMS. A mild nonfluent patient increased from a three-word 
phrase length, to a five-word phrase length (Cookie Theft picture, BDAE); and a moderate nonfluent patient increased from a one-word phrase length, to a three-word phrase length.

Similar positive results have been observed with another mild nonfluent aphasia patient who started TMS treatments at 2.4 yr. poststroke. ${ }^{36}$ After determination of this patient's "best response" RH ROI to suppress from Phase 1 TMS (see Phase 1 TMS results for this case, Figure 1), the patient entered and completed Phase 2. At 3 and 6 months post- Phase 2 TMS treatments, this patient's BNT scores improved significantly (>2 SD) from baseline (Table 1). At 27 months post-TMS (4.6 years poststroke), his increase on the BNT remained significant compared to baseline. An improvement in longest phrase length (Cookie Theft picture, BDAE) was also observed in this patient, post- TMS. See Table 1 for language data and speech samples for his spontaneous speech pre- and post- Phase 2 TMS. He received no individual speech therapy during, or post- TMS. Another chronic nonfluent aphasia patient treated at 7 years poststroke using the same TMS protocol, also showed increased phrase length and complexity in elicited, propositional speech at 2, 6 and 10 months post-TMS. ${ }^{49}$

\section{1d. Functional MRI, Pre- and Post- TMS}

Functional MRI (fMRI) has been used to examine brain activation during overt naming, preand post- a two-week TMS treatment series (Phase 2 TMS) in two chronic nonfluent aphasia patients. ${ }^{50}$ One patient (P1) improved in naming, and phrase length in propositional speech, lasting at least 2 years post- TMS. The other patient (P2) showed no change in naming or propositional speech post- TMS.

For P1, language was tested at 2, 6, 16, and 43 months post- Phase 2 TMS. He had significant improvement on the BNT, and longest phrase length (Cookie Theft picture, BDAE). Auditory comprehension was largely unchanged. See Table 2a.

For P2, pre- TMS language testing was performed at baseline (1.5 years poststroke), and at 2 and 6 months post- Phase 2 TMS. His spontaneous speech consisted primarily of only stereotypies. He had no change on the BNT or in longest phrase length (Cookie Theft picture, BDAE). He improved by 2 SD post- TMS on BDAE Auditory comprehension subtests for Commands at 2 and 6 months post- TMS and Complex Ideational Material at 6 months. See Table $2 b$.

Results from this overt naming fMRI study by Martin and colleagues, ${ }^{50}$ showed at pre- TMS (as well as at 3 months and at 16 months post- TMS), that $\mathrm{P} 1$ had activation in $\mathrm{R}$ and $\mathrm{L}$ sensorimotor cortex (mouth area), R IFG, and in R and L SMA. At 16 months post- TMS, however, there was a significant increase in activation in the L SMA, compared to pre-, and to 3 months post- TMS ( $p<.02 ; \mathrm{p}<.05$, respectively). There was also a trend towards significantly greater activation in L SMA than R SMA at 16 months and 46 months postTMS ( $p<.08 ; \mathrm{p}<.09$, respectively). Pre- TMS there had been no difference between $\mathrm{L}$ and $\mathrm{R}$ SMA activation. A shift to stronger L SMA activation was first observed at 16 months postTMS. At this time, his highest accuracy rate for picture naming (58\%) was observed, compared to only $28 \%$ pre- TMS and $42 \%$ at 3 months post- TMS. There were no intervening overt speech fMRI scans between 3 and 16 months post- TMS. The new LH activation remained present, even at 46 months post- TMS (nearly 4 years post- TMS) when the patient was almost 14 years poststroke.

Results from overt naming fMRI with P2, showed that pre- TMS, significant activation in $\mathrm{R}$ IFG was present and he named only $3 \%$ of the pictures. At 3 and 6 months post- TMS, there was no longer significant activation in R IFG (the area suppressed with $1 \mathrm{~Hz}$ rTMS), but significant activation was present in R sensorimotor cortex. Although P2 had significant activation in both the L and R SMA on all three fMRI scans (pre- TMS, and at 3 and 6 
months post-TMS), ROI analyses showed no difference across sessions in the L or R SMA activation. ${ }^{50}$ In addition, suppression of R PTr with rTMS resulted in no new, lasting perilesional $\mathrm{LH}$ activation across sessions. His naming remained only at 1-2 pictures during all three fMRI scans. His BNT score and longest phrase length remained at 1 word, postTMS.

Lesion site likely played a role in each patient's fMRI activation pattern, and level of response to TMS treatment. P2 had an atypical frontal lesion in the L motor and premotor cortex that extended high, near brain vertex, with deep white matter lesion near L SMA. Additionally, $\mathrm{P} 2$ had lesion in the posterior middle frontal gyrus at the junction of the premotor cortex, an area important for naming. ${ }^{51} \mathrm{P} 2$ also had lesion located inferior and posterior to Wernicke's area, in BA 21 and 37. P1 had no lesion in these three areas.

The significant increase in activation of the L SMA post- TMS in P1, who improved in naming, is compatible with previous fMRI studies that observed new L SMA activation to be present in aphasia patients with better outcome. ${ }^{16,17}$ This improved LH activation is also compatible with previous studies that have observed better outcome following language therapy to be associated with new, perilesional LH activation. ${ }^{21-24}$

Based on their results, Martin and colleagues ${ }^{50}$ have suggested minimum criteria for entry into TMS treatment as follows: 1) the patient should have a mean score of at least three pictures named correctly on the BNT (without phonemic cueing), tested across three test sessions; 2) the patient should not produce stereotypies in spontaneous speech. P2 would not have met these minimum criteria for entry, as his mean BNT score across three test sessions pre-TMS was only 1.67 ( $\mathrm{SD}=1.15$, range, $1-3)$; and he produced stereotypies during spontaneous speech. One severe nonfluent, global aphasia patient with a 1-word phrase length, but who did not produce stereotypies, had a good response to the TMS protocol of Naeser et al. ${ }^{37}$ She named 4 pictures on the BNT pre- TMS; 7 pictures, at 2 months postTMS; and 12 pictures, at 8 months post- TMS. The degree of improvement resulted in the patient's ability to have individual speech therapy sessions, leading to continued improvement in naming and communication.

\section{Part 2. TMS plus Constraint-Induced Language Therapy (CILT)}

\section{2a. Background and Rationale}

CILT is an intensive speech therapy program shown to significantly improve naming, following a series of 10 CILT treatments. ${ }^{52-54}$ During CILT, patients are only allowed to respond with verbal naming for a picture (no gestures or writing or sound effects are permitted). An opaque screen is placed on a table where the Speech-Language Pathologist (SLP) is seated on one side, and the patient, on the other. There is eye contact above the screen, but it is not possible for the patient to use hand gestures or writing to communicate. For example, the patient may be given a series of picture cards, and he must communicate verbally to the therapist, which card he has on his side of the screen and/or ask the SLP if there is a similar card on the other side of the screen. The response required from the patient is gradually increased from single words, up to phrases and even sentences.

Results from the Maher and colleagues CILT study ${ }^{52}$ showed improvement in naming (BNT), primarily at 1 month follow-up testing (not immediately post- CILT). Their results on the Western Aphasia Battery, Aphasia Quotient, had showed improvement immediately post- CILT and also at 1 month follow-up. Maher and colleagues ${ }^{52}$ suggest that "...the impact of CILT may continue to be active beyond the direct treatment period." The impact of TMS also continues to be active beyond the direct treatment period - e.g. at least 2 
months or more post-TMS. ${ }^{33,36,37,49,50}$ Combining these two forms of therapy (TMS+CILT) may promote maximum gains in naming for chronic aphasia patients.

\section{2b. TMS plus CILT Treatment Protocol with Nonfluent Aphasia}

Patients who have completed Phase 1 and Phase 2 TMS, ${ }^{33}$ with good response, were eligible to enter a study by Naeser and colleagues that combined TMS+CILT. ${ }^{55}$ The "best response" RH ROI that was suppressed during Phase 2 TMS, was suppressed in the same manner - i.e., $1 \mathrm{~Hz}$ rTMS for $20 \mathrm{~min}, 90 \%$ MT for 10 sessions over a two-week period (weekdays only). A 3-hour CILT session immediately followed each 20-min TMS session.

A severe nonfluent aphasia patient, who initially received only the Phase 1 and Phase 2 TMS protocol at 6.5 years poststroke, later participated in TMS+CILT ${ }^{55}$ This patient participated in TMS+CILT at 12.5 years poststroke ( 5 years, 10 months after the initial TMS series). Prior to TMS+CILT, her object naming ability was tested on a set of 250 color pictures, 3 times. One-third of the color pictures presented as stimulus items for therapy had always been named on pre-testing (3/3); one-third, she had sometimes named (1-2/3); and one-third, she had never named (0/3). During CILT, 6 pictures were presented at a time (2 pictures had always been named at entry pre-testing; 2 , sometimes; 2 , never). A total of 18 pictures were presented during CILT each day ( 3 sets of 6 pictures each).

Language outcome measures included the BNT and subtests on the BDAE. These tests were administered at baseline pre-TMS ( 3 times); and at 1 and 6 months post- the 10th treatment in the TMS+CILT protocol by Naeser and colleagues. ${ }^{55}$ Significant improvement was defined as $>2$ SD above baseline.

\section{Naming Probe Testing}

To examine changes that might occur during intervention, Naming Probe Testing (NPT) was also completed. BDAE naming subtests (Actions, Animals, Tools/Implements), the BNT, and the action naming pictures from Druks and Masterson ${ }^{56}$ were administered 12 times pre- TMS (including the 3 baseline testings). In addition, immediately post- each CILT session, daily NPT was administered (10 times). After the $10^{\text {th }}$ treatment, NPT was again administered (10 separate times). The time-series data for each test were later analyzed using a double bootstrap method (http://www.stat.wmich.edu/slab/Software/ Timeseries.html). ${ }^{57}$

\section{2c. Results for TMS plus CILT}

On the primary language outcome measures, this severe nonfluent aphasia patient improved $>2$ SD on BDAE Action Naming, Tools/Implements, and Single Word Repetition. Improvement in BDAE Action Naming was only observed following the second TMS series, where CILT was included. See Fig. 2. These results suggest that additional improvements may be gained when TMS is combined with language therapy such as CILT, in chronic stroke patients with aphasia. On NPT, the time-series analysis showed significant improvement on BDAE Action Naming ( $\mathrm{p}=.035)$; and Tools/Implements $(\mathrm{p}=.010)$. There was a trend toward significant improvement on the action naming pictures from Druks and Masterson $^{56}$ (mean pre-TMS, 6; SD, 1.48; range 3-7, and mean post-TMS, 7.90; SD, 1.73, range $6-10, p=.308$ ). 


\section{Part 3. Possible mechanisms, and new TMS studies in Aphasia}

\section{3a. Possible Mechanisms}

The mechanisms associated with language improvement post- TMS treatments in chronic stroke patients with aphasia are unknown. The presence of a differential effect on naming following suppression of R PTr (facilitation of naming) vs. suppression of R POp (impairment of naming) during Phase 1 by Naeser and colleagues ${ }^{43}$ in nonfluent aphasia patients may provide some insight regarding the potential role of R POp in aphasia. This differential effect suggests potential for different pathways with posterior language regions (temporo-parietal regions), and different roles for the R PTr vs. R POp.

For example, in recent DTI studies ${ }^{58-60}$ white matter pathways were observed to follow a more dorsal route between L posterior Broca's area (likely POp; and premotor cortices) and anterior supramarginal gyrus (SMG), via the arcuate fasiculus (AF). However, major pathways between L anterior Broca's area (likely PTr, ventrolateral prefrontal cortex), and L superior temporal gyrus have been observed to follow a more ventral route via the extreme capsule (not via the AF). ${ }^{58,59}$ The primary role for the dorsal route in the LH is mainly restricted to sensory-motor mapping of sound to articulation, and higher-order articulatory control of speech ${ }^{58}$, where the POp is connected directly with premotor area 6 (involved with orofacial musculature) ${ }^{61-62}$ The ventral route in the LH likely performs linguistic processing of sound to meaning, requiring temporo-frontal interaction and top-down regulation of linguistic processing such as verbal retrieval ${ }^{62}$, and lexical/semantic aspects of language processing. ${ }^{59,62-67}$ In support of this, a dissociation between the roles of L PTr and L POp in semantic versus phonological tasks has been supported by TMS application to these two areas in normals, where differential/opposite effects were observed. ${ }^{68}$

Kaplan and colleagues recently studied white matter pathways for the POp and PTr in the $\mathrm{RH}$. Their study observed similar white matter connections in the RH, to those reported above in the LH. ${ }^{69}$ In most cases, there were no direct pathways between R PTr and R AF; but in most cases, direct pathways were present between R POp and R AF. See Figure 3. The presence of these different RH pathways may support the differential effect on naming, following suppression of R PTr vs. R POp, in nonfluent aphasia cases. Suppression of R POp with rTMS may have had a direct, negative effect on the phonological aspects of naming and higher-order articulatory control of speech in the nonfluent aphasia patients.

Suppression of R PTr with $1 \mathrm{~Hz}$ rTMS may promote less inhibition of R POp from R PTr, via U-fibers. Better modulation of R POp may also indirectly support better modulation of $\mathrm{R}$ ventral premotor cortex (vPMC), located one gyrus posterior to POp. A contributory role for the R POp and the R vPMC in aphasia recovery has been posited since the time of Barlow (1877). ${ }^{70}$ Current rTMS data support a contributory role for R POp in aphasia recovery because suppression of R POp impaired naming. In fact, Naeser and colleagues have never observed the R POp to be a "best response" ROI to improve naming. ${ }^{33,36,37,43}$

Finally, it is worth considering that the POp and vPMC are part of the bilateral, mirror neuron system. ${ }^{71}$ Mirror neurons are cells that fire during both production and perception of similar actions. They are important in child language acquisition ${ }^{72}$ and they are thought to be present bilaterally. The R POp and R vPMC may have relevance in promoting recovery in aphasia (especially in phonological and motor aspects of speech), due to the presence of mirror neurons. This could help to clarify why suppression of R POp in nonfluent aphasia patients impaired naming, - i.e., there was possibly interrupted activation of some mirror neurons in this area; however, this is unknown and the unique role of R POp and vPMC in aphasia recovery requires further study. 


\section{3b. New TMS Studies in Aphasia}

Recent research by Barwood and colleagues ${ }^{73}$ utilized rTMS to suppress the apical portion of R PTr for two weeks in chronic nonfluent aphasia patients, in a manner similar to the Phase 2 rTMS treatment protocol by Naeser et al. ${ }^{33,36,37}$ Similar results were observed. Significant improvements were observed in BDAE naming Actions, BDAE naming Tools/ Implements, BDAE overall score and BDAE picture description at 2 months post- real rTMS treatment. Importantly, these investigators found no significant improvements at 2 months post- sham rTMS.

Weiduschat et al., $2011^{74}$, obtained PET scans before and after rTMS combined with conventional speech therapy, in a variety of subacute stroke patients with aphasia. Language results showed a significant improvement in total Aachen Aphasia Test scores in cases who received rTMS to suppress the R PTr, plus speech therapy, versus those who received control rTMS to suppress the vertex, plus speech therapy. PET results showed that cases who had received the control rTMS retained a $\mathrm{R}$ lateralization of brain activation during verb generation; whereas those cases who received rTMS over R PTr, no longer retained R lateralization and there was greater improvement in language.

In summary, the TMS studies by Naeser and colleagues, ${ }^{33,36,37,49}$ as well as these new TMS studies $^{73,74}$, all suggest that use of $1 \mathrm{~Hz}$ rTMS for a series of at least 10 rTMS treatments, results in significant improvement in naming; and often in phrase length during propositional speech. These improvements are long-lasting, up to 2 months, or even as long as 2 years, post- TMS. ${ }^{33,36,37,49,50}$ Functional imaging studies report significant improvement in naming in those cases with new $\mathrm{LH}$ activation ${ }^{50}$ or a shift to overall $\mathrm{LH}$ lateralization. ${ }^{74}$ When TMS is combined with speech therapy, additional improvement has been observed, beyond TMS alone. ${ }^{55,74}$ Additional TMS studies in aphasia are likely to replicate, and expand these findings.

\section{Acknowledgments}

The authors thank Anna Kharaz for assistance with the manuscript.

Research supported by NIH grant RO1 DC05672 from the National Institute on Deafness and Other Communication Disorders (NIDCD), Bethesda, MD, and a grant from the Medical Research Service, Department of Veterans Affairs, Washington, D.C. (to M.A.N.); a K24 NIH award (RRO18875, to A.P.-L) and the HarvardThorndike General Clinical Research Center (NCRR MO1 RR01032); and Harvard Clinical and Translational Science Center (UL1 RR025758); and a P30 DC05207 NIDCD grant to the Harold Goodglass BU Aphasia Research Center. Clinical Trials Registration Number: NCT00608582

Financial Disclosure: No party having a direct interest in the results of the research supporting this article has or will confer a benefit on the authors or on any organization with which they are associated The authors certify that all financial and material support for this research (eg, NIH or NHS grants) and work are clearly identified in the title page of the manuscript.

\section{Abbreviations}

$\begin{array}{ll}\text { TMS } & \text { Transcranial magnetic stimulation } \\ \text { rTMS } & \text { Repetitive transcranial magnetic stimulation } \\ \text { CILT } & \text { Constraint-Induced Language Therapy } \\ \text { RH } & \text { right hemisphere } \\ \text { LH } & \text { left hemisphere } \\ \text { SMA } & \text { supplementary motor area }\end{array}$




$\begin{array}{ll}\text { BA } & \text { Brodmann area } \\ \text { IFG } & \text { inferior frontal gyrus } \\ \text { ROI } & \text { region of interest } \\ \text { R } & \text { right } \\ \text { L } & \text { left } \\ \text { BDAE } & \text { Boston Diagnostic Aphasia Exam } \\ \text { BNT } & \text { Boston Naming Test } \\ \text { S\&V } & \text { Snodgrass \& Vanderwart } \\ \text { RT } & \text { response time } \\ \text { min } & \text { minutes } \\ \text { MT } & \text { motor threshold } \\ \text { L FDI } & \text { left first dorsal interosseus muscle } \\ \text { MEP } & \text { motor evoked potential } \\ \text { POp } & \text { pars opercularis } \\ \text { PTr } & \text { pars triangularis } \\ \text { MRI } & \text { magnetic resonance imaging } \\ \text { fMRI } & \text { functional MRI } \\ \text { SLP } & \text { Speech-Language Pathologist } \\ \text { NPT } & \text { Naming Probe Testing } \\ \text { SMG } & \text { supramarginal gyrus } \\ \text { AF } & \text { arcuate fasciculus } \\ \text { vPMC } & \text { ventral pre-motor cortex } \\ \text { PET } & \text { positron emission tomography } \\ \end{array}$

\section{References}

1. Barker AT, Jalinous R, Freeston IL. Non-invasive magnetic stimulation of human motor cortex. Lancet. 1985; 1:1106-1107. [PubMed: 2860322]

2. Lefaucheur JP. Stroke recovery can be enhanced by using repetitive transcranial magnetic stimulation (rTMS). Neurophysiol Clin. 2006; 36:105-115. [PubMed: 17046605]

3. Pascual-Leone, A.; Davey, N.; Wasserman, EM.; Rothwell, J.; Puri, B., editors. Handbook of Transcranial Magnetic Stimulation. London, UK: Arnold Press; 2002.

4. Wasserman, EM.; Epstein, CM.; Ziemann, U.; Walsh, V.; Paus, T.; Lisanby, SH., editors. The Oxford Handbook of Transcranial Stimulation. New York: Oxford University Press; 2008.

5. Rothwell JC. Techniques and mechanisms of action of transcranial stimulation of the human motor cortex. J Neurosci Methods. 1997; 74:113-122. [PubMed: 9219881]

6. Pascual-Leone A, Tormos JM, Keenan J, Tarazona F, Canete C, Catala MD. Study and modulation of human cortical excitability with transcranial magnetic stimulation. J Clin Neurophysiol. 1998; 15:333-343. [PubMed: 9736467]

7. Chen R, Classen J, Gerloff C, et al. Depression of motor cortex excitability by low-frequency transcranial magnetic stimulation. Neurology. 1997; 48:1398-1403. [PubMed: 9153480] 
8. Maeda F, Keenan JP, Tormos JM, Topka H, Pascual-Leone A. Modulation of corticospinal excitability by repetitive transcranial magnetic stimulation. Clin Neurophysiol. 2000; 111:800-805. [PubMed: 10802449]

9. Pascual-Leone A, Valls-Sole J, Wassermann EM, Hallett M. Responses to rapid-rate transcranial magnetic stimulation of the human motor cortex. Brain. 1994; 117(Pt 4):847-858. [PubMed: 7922470]

10. Belin P, Van Eeckhout P, Zilbovicius M, et al. Recovery from nonfluent aphasia after melodic intonation therapy: a PET study. Neurology. 1996; 47:1504-1511. [PubMed: 8960735]

11. Martin PI, Naeser MA, Doron KW, Bogdan A, Baker EH, Kurland J, Renshaw P, Yurgelun-Todd D. Overt naming in aphasia studied with a functional MRI hemodynamic delay design. Neuroimage. 2005; 28:194-204. [PubMed: 16009568]

12. Naeser MA, Martin PI, Baker EH, Hodge SM, Sczerzenie SE, Nicholas M, Palumbo CL, Goodglass H, Wingfield A, Samaraweera R, Harris G, Baird A, Renshaw P, Yurgelun-Todd D. Overt propositional speech in chronic nonfluent aphasia studied with the dynamic susceptibility contrast fMRI method. Neuroimage. 2004; 22:29-41. [PubMed: 15109995]

13. Perani D, Cappa SF, Tettamanti M, Rosa M, Scifo P, Miozzo A, Basso A, Fazio F. A fMRI study of word retrieval in aphasia. Brain Lang. 2003; 85:357-368. [PubMed: 12744947]

14. Rosen HJ, Petersen SE, Linenweber MR, Snyder AZ, White DA, Chapman L, Dromerick AW, Fiez JA, Corbetta MD. Neural correlates of recovery from aphasia after damage to left inferior frontal cortex. Neurology. 2000; 55:1883-1894. [PubMed: 11134389]

15. Price CJ, Crinion J. The latest on functional imaging studies of aphasic stroke. Curr Opin Neurol. 2005; 18:429-434. [PubMed: 16003120]

16. Karbe H, Thiel A, Weber-Luxenburger G, Herholz K, Kessler J, Heiss WD. Brain plasticity in poststroke aphasia: what is the contribution of the right hemisphere? Brain Lang. 1998; 64:215230. [PubMed: 9710490]

17. Heiss WD, Thiel A. A proposed regional hierarchy in recovery of post-stroke aphasia. Brain Lang. 2006; 98:118-123. [PubMed: 16564566]

18. Saur D, Lange R, Baumgaertner A, Schraknepper V, Willmes K, Rijntjes M, Weiller C. Dynamics of language reorganization after stroke. Brain. 2006; 129:1371-1384. [PubMed: 16638796]

19. Hillis AE, Kleinman JT, Newhart M, Heidler-Gary J, Gottesman R, Barker PB, Aldrich E, Llinas R, Wityk R, Chaudhry P. Restoring cerebral blood flow reveals neural regions critical for naming. J Neurosci. 2006; 26:8069-8073. [PubMed: 16885220]

20. Winhuisen L, Thiel A, Schumacher B, Kessler J, Rudolf J, Haupt WF, Heiss WD. Role of the contralateral inferior frontal gyrus in recovery of language function in poststroke aphasia: a combined repetitive transcranial magnetic stimulation and positron emission tomography study. Stroke. 2005; 36:1759-1763. [PubMed: 16020770]

21. Small SL, Flores DK, Noll DC. Different neural circuits subserve reading before and after therapy for acquired dyslexia. Brain Lang. 1998; 62:298-308. [PubMed: 9576825]

22. Leger A, Demonet JF, Ruff S, Aithamon B, Touyeras B, Puel M, Boulanouar K, Cardebat D. Neural substrates of spoken language rehabilitation in an aphasic patient: an fMRI study. Neuroimage. 2002; 17:174-183. [PubMed: 12482075]

23. Cornelissen K, Laine M, Tarkiainen A, Jarvensivu T, Martin N, Salmelin R. Adult brain plasticity elicited by anomia treatment. J Cogn Neurosci. 2003; 15:444-461. [PubMed: 12729495]

24. Richter M, Miltner WH, Straube T. Association between therapy outcome and right-hemispheric activation in chronic aphasia. Brain. 2008; 131:1391-1401. [PubMed: 18349055]

25. Cherney LR, Small SL. Task-dependent changes in brain activation following therapy for nonfluent aphasia: discussion of two individual cases. J Int Neuropsychol Soc. 2006; 12:828-842. [PubMed: 17064446]

26. Crosson B, Moore AB, Gopinath K, White KD, Wierenga CE, Gaiefsky ME, Fabrizio KS, Peck KK, Soltysik D, Milsted C, Briggs RW, Conway TW, Gonzalez Rothi LJ. Role of the right and left hemispheres in recovery of function during treatment of intention in aphasia. J Cogn Neurosci. 2005; 17:392-406. [PubMed: 15814000] 
27. Peck KK, Moore AB, Crosson BA, Gaiefsky M, Gopinath KS, White K, Briggs RW. Functional magnetic resonance imaging before and after aphasia therapy: shifts in hemodynamic time to peak during an overt language task. Stroke. 2004; 35:554-559. [PubMed: 14739418]

28. Raboyeau G, De Boissezon X, Marie N, Balduyck S, Puel M, Bezy C, Demonet JF, Cardebat D. Right hemisphere activation in recovery from aphasia: lesion effect or function recruitment? Neurology. 2008; 70:290-298. [PubMed: 18209203]

29. Fernandez B, Cardebat D, Demonet JF, Joseph PA, Mazaux JM, Barat M, Allard M. Functional MRI follow-up study of language processes in healthy subjects and during recovery in a case of aphasia. Stroke. 2004; 35:2171-2176. [PubMed: 15297629]

30. Gold BT, Kertesz A. Right hemisphere semantic processing of visual words in an aphasic patient: an fMRI study. Brain Lang. 2000; 73:456-465. [PubMed: 10860566]

31. Thompson CK. Neuroplasticity: evidence from aphasia. J Commun Disord. 2000; 33:357-366. [PubMed: 11001162]

32. Crosson B, McGregor K, Gopinath KS, Conway TW, Benjamin M, Chang YL, Moore AB, Raymer AM, Briggs RW, Sherod MG, Wierenga CE, White KD. Functional MRI of language in aphasia: a review of the literature and the methodological challenges. Neuropsychol Rev. 2007; 17:157-177. [PubMed: 17525865]

33. Naeser MA, Martin PI, Nicholas M, Baker EH, Seekins H, Kobayashi M, Theoret H, Fregni F, Maria-Tormos J, Kurland J, Doron KW, Pascual-Leone A. Improved picture naming in chronic aphasia after TMS to part of right Broca's area: an open-protocol study. Brain Lang. 2005; 93:95105. [PubMed: 15766771]

34. Kapur N. Paradoxical functional facilitation in brain-behaviour research. A critical review. Brain. 1996; 119(Pt):1775-1790. [PubMed: 8931597]

35. Theoret H, Kobayashi M, Valero-Cabre A, Pascual-Leone A. Exploring paradoxical functional facilitation with TMS. Suppl Clin Neurophysiol. 2003; 56:211-219. [PubMed: 14677397]

36. Naeser MA, Martin PI, Lundgren K, Klein R, Kaplan J, Treglia E, Ho M, Nicholas M, Alonso M, Pascual-Leone A. Improved Language in a Chronic Nonfluent Aphasia Patient After Treatment With CPAP and TMS. Cogn Behav Neurol. 2010; 23:29-38. [PubMed: 20299861]

37. Naeser MA, Martin PI, Nicholas M, Baker EH, Seekins H, Helm-Estabrooks N, Cayer-Meade C, Kobayashi M, Theoret H, Fregni F, Tormos JM, Kurland J, Doron KW, Pascual-Leone A. Improved naming after TMS treatments in a chronic, global aphasia patient--case report. Neurocase. 2005; 11:182-193. [PubMed: 16006338]

38. Tassinari CA, Cincotta M, Zaccara G, Michelucci R. Transcranial magnetic stimulation and epilepsy. Clin Neurophysiol. 2003; 114:777-798. [PubMed: 12738425]

39. Goodglass, H.; Kaplan, E. Assessment of Aphasia and Related Disorders. Philadelphia, PA: Lea and Febiger; 1983.

40. Goodglass, H.; Kaplan, E.; Barresi, B. The Assessment of Aphasia and Related Disorders. 3rd Ed. Philadelphia, PA: Lippincott, Williams and Wilkins; 2001.

41. Kaplan, E.; Goodglass, H.; Weintraub, S. The Boston Naming Test. 3rd Ed. Philadelphia, PA: Lippincott, Williams and Wilkins; 2001.

42. Snodgrass JG, Vanderwart M. A standardized set of 260 pictures: norms for name agreement, image agreement, familiarity, and visual complexity. J Exp Psychol [Hum Learn]. 1980; 6:174215.

43. Naeser, MA.; Martin, P.; Fregni, F.; Theoret, H.; Kobayashi, M.; Nicholas, M.; Baker, E.; MariaTormos, J.; Steven, M.; Pascual-Leone, A. Modulation of cortical areas with repetitive transcranial magnetic stimulation to improve naming in nonfluent aphasia [Abstract\#133]. NeuroImage; Proceedings of the 8th International Conference on Functional Mapping of the Human Brain; Sendai, Japan. 2002 June 2-6; 2002.

44. Wassermann EM. Risk and safety of repetitive transcranial magnetic stimulation: report and suggested guidelines from the International Workshop on the Safety of Repetitive Transcranial Magnetic Stimulation, June 5-7, 1996. Electroencephalogr Clin Neurophysiol. 1998; 108:1-16. [PubMed: 9474057] 
45. Rossi S, Hallett M, Rossini PM, Pascual-Leone A. Safety, ethical considerations, and application guidelines for the use of transcranial magnetic stimulation in clinical practice and research. Clin Neurophysiol. 2009; 120:2008-2039. [PubMed: 19833552]

46. Machii K, Cohen D, Ramos-Estebanez C, Pascual-Leone A. Safety of rTMS to non-motor cortical areas in healthy participants and patients. Clin Neurophysiol. 2006; 117:455-471. [PubMed: 16387549]

47. Amunts K, Schleicher A, Burgel U, Mohlberg H, Uylings HB, Zilles K. Broca's region revisited: cytoarchitecture and intersubject variability. J Comp Neurol. 1999; 412:319-341. [PubMed: 10441759]

48. Amunts K, Weiss PH, Mohlberg H, Pieperhoff P, Eickhoff S, Gurd JM, Marshall JC, Shah NJ, Fink GR, Zilles K. Analysis of neural mechanisms underlying verbal fluency in cytoarchitectonically defined stereotaxic space--the roles of Brodmann areas 44 and 45. Neuroimage. 2004; 22:42-56. [PubMed: 15109996]

49. Hamilton RH, Sanders L, Benson J, Faseyitan O, Norise C, Naeser M, Martin P, Coslett HB. Stimulating conversation: enhancement of elicited propositional speech in a patient with chronic non-fluent aphasia following transcranial magnetic stimulation. Brain Lang. 2010; 113:45-50. [PubMed: 20159655]

50. Martin PI, Naeser MA, Ho M, Doron KW, Kurland J, Kaplan J, Wang Y, Nicholas M, Baker EH, Fregni F, Pascual-Leone A. Overt naming fMRI pre- and post-TMS: Two nonfluent aphasia patients, with and without improved naming post-TMS. [published erratum in: Brain Lang 2010;112(2):135 Alonso, Miguel- added]. Brain Lang. 2009; 111:20-35. [PubMed: 19695692]

51. Duffau H, Capelle L, Denvil D, Gatignol P, Sichez N, Lopes M, Sichez JP, Van Effenterre R. The role of dominant premotor cortex in language: a study using intraoperative functional mapping in awake patients. Neuroimage. 2003; 20:1903-1914. [PubMed: 14683696]

52. Maher LM, Kendall D, Swearengin JA, Rodriguez A, Leon SA, Pingel K, Holland A, Rothi LJ. A pilot study of use-dependent learning in the context of Constraint Induced Language Therapy. J Int Neuropsychol Soc. 2006; 12:843-852. [PubMed: 17064447]

53. Meinzer M, Djundja D, Barthel G, Elbert T, Rockstroh B. Long-term stability of improved language functions in chronic aphasia after constraint-induced aphasia therapy. Stroke. 2005; 36:1462-1466. [PubMed: 15947279]

54. Pulvermuller F, Neininger B, Elbert T, Mohr B, Rockstroh B, Koebbel P, Taub E. Constraintinduced therapy of chronic aphasia after stroke. Stroke. 2001; 32:1621-1626. [PubMed: 11441210]

55. Naeser MA, Martin PI, Treglia E, Ho M, Kaplan E, Bashir S, Hamilton R, Coslett HB, PascualLeone A. Research with rTMS in the treatment of aphasia. Restor Neurol Neurosci. 2010; 28:511529. [PubMed: 20714075]

56. Druks, J.; Masterson, J. An Object and Action Naming Battery. >Hove, East Sussex, UK: Psychology Press Ltd; 2000.

57. McKnight SD, McKean JW, Huitema BE. A double bootstrap method to analyze linear models with autoregressive error terms. Psychol Methods. 2000; 5:87-101. Computer software available from: URL: http://www.stat.wmich.edu/slab/Software/Timeseries.html. [PubMed: 10937324]

58. Frey S, Campbell JS, Pike GB, Petrides M. Dissociating the human language pathways with high angular resolution diffusion fiber tractography. J Neurosci. 2008; 28:11435-11444. [PubMed: 18987180]

59. Saur D, Kreher BW, Schnell S, Kummerer D, Kellmeyer P, Vry MS, Umarova R, Musso M, Glauche V, Abel S, Huber W, Rijntjes M, Hennig J, Weiller C. Ventral and dorsal pathways for language. Proc Natl Acad Sci U S A. 2008; 105:18035-18040. [PubMed: 19004769]

60. Croxson PL, Johansen-Berg H, Behrens TE, Robson MD, Pinsk MA, Gross CG, Richter W, Richter MC, Kastner S, Rushworth MF. Quantitative investigation of connections of the prefrontal cortex in the human and macaque using probabilistic diffusion tractography. J Neurosci. 2005; 25:8854-8866. [PubMed: 16192375]

61. Petrides M, Cadoret G, Mackey S. Orofacial somatomotor responses in the macaque monkey homologue of Broca's area. Nature. 2005; 435:1235-1238. [PubMed: 15988526] 
62. Petrides, M. Broca's area in the human and the non-human primate brain. In: Grodzinsky, Y.; Amunts, K., editors. Broca's Region. New York: Oxford University Press; 2006.

63. Devlin JT, Matthews PM, Rushworth MF. Semantic processing in the left inferior prefrontal cortex: a combined functional magnetic resonance imaging and transcranial magnetic stimulation study. J Cogn Neurosci. 2003; 15:71-84. [PubMed: 12590844]

64. Gold BT, Buckner RL. Common prefrontal regions coactivate with dissociable posterior regions during controlled semantic and phonological tasks. Neuron. 2002; 35:803-812. [PubMed: 12194878]

65. Nixon P, Lazarova J, Hodinott-Hill I, Gough P, Passingham R. The inferior frontal gyrus and phonological processing: an investigation using rTMS. J Cogn Neurosci. 2004; 16:289-300. [PubMed: 15068598]

66. Poldrack RA, Wagner AD, Prull MW, Desmond JE, Glover GH, Gabrieli JD. Functional specialization for semantic and phonological processing in the left inferior prefrontal cortex. Neuroimage. 1999; 10:15-35. [PubMed: 10385578]

67. Price CJ, Wise RJ, Warburton EA, Moore CJ, Howard D, Patterson K, Frackowiak RS, Friston KJ. Hearing and saying. The functional neuro-anatomy of auditory word processing. Brain. 1996; 119(Pt 3):919-931. [PubMed: 8673502]

68. Gough PM, Nobre AC, Devlin JT. Dissociating linguistic processes in the left inferior frontal cortex with transcranial magnetic stimulation. J Neurosci. 2005; 25:8010-8016. [PubMed: 16135758]

69. Kaplan E, Naeser MA, Martin PI, Ho M, Wang Y, Baker E, Pascual-Leone A. Horizontal portion of arcuate fasciculus fibers track to pars opercularis, not pars triangularis, in right and left hemispheres: a DTI study. Neuroimage. 2010; 52:436-444. [PubMed: 20438853]

70. Barlow T. Brit Med J. 1877:103.

71. Iacoboni M. The role of premotor cortex in speech perception: evidence from fMRI and rTMS. J Physiol Paris. 2008; 102:31-34. [PubMed: 18440208]

72. Rizzolatti G, Craighero L. The mirror-neuron system. Annu Rev Neurosci. 2004; 27:169-192. [PubMed: 15217330]

73. Barwood $\mathrm{CH}$, Murdoch BE, Whelan BM, et al. Improved language performance subsequent to low-frequency rTMS in patients with chronic non-fluent aphasia post-stroke. Eur J Neurol. 2010

74. Weiduschat N, Thiel A, Rubi-Fessen I, et al. Effects of repetitive transcranial magnetic stimulation in aphasic stroke: a randomized controlled pilot study. Stroke. 2011; 42:409-415. [PubMed: 21164121] 


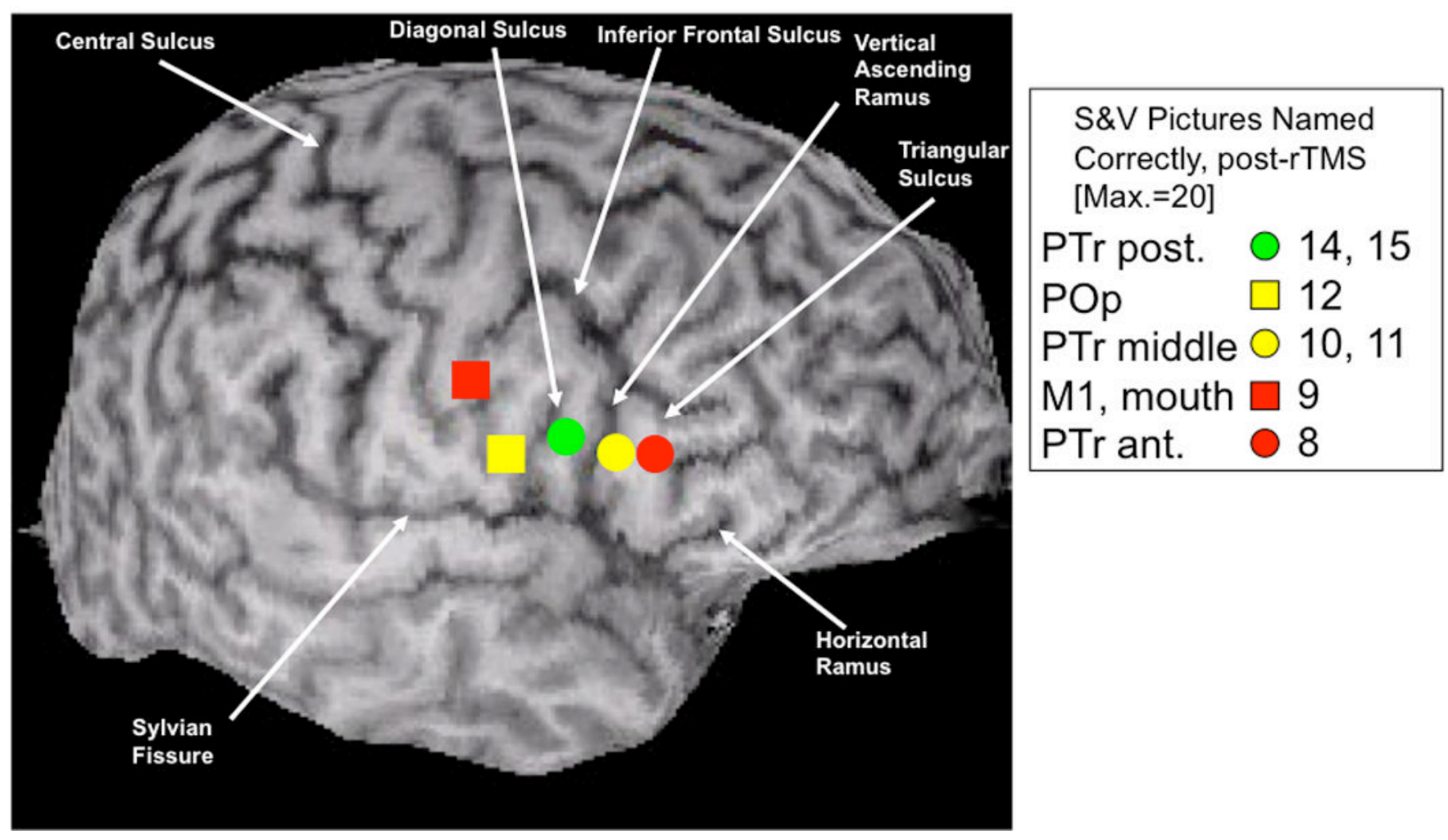

Figure 1.

Legend box shows naming data for a single aphasia case, acquired immediately after suppression of 5 different RH cortical ROIs during exploratory Phase 1 TMS. Location of five frontal, RH ROIs are shown where each was suppressed in separate TMS sessions, with $1 \mathrm{~Hz}$ rTMS for $10 \mathrm{~min}$. These five ROIs included R M1, mouth (orbicularis oris muscle, as verified with MEP), and four subregions within $\mathrm{R}$ Broca's area as defined in the text, using sulcal boundaries (arrows). A diagonal sulcus was present in the $\mathrm{RH}$ in this case. The PTr posterior ROI (green symbol), was the best-response ROI - i.e., the area associated with a naming score that reached at least 2 SD above baseline S\&V naming ability (e.g., 15). During Phase 2 TMS, the PTr posterior ROI (green symbol) was used as the target for suppression with $1 \mathrm{~Hz}$ rTMS for ten, 20-min treatments in this case. Note that the number of pictures named correctly immediately post- rTMS decreased for any given ROI, as the distance from the best-response ROI increased by 1 or $2 \mathrm{~cm}$, in an anterior or posterior direction. ROI, region of interest; PTr, pars triangularis; POp, pars opercularis; S\&V, Snodgrass and Vanderwart. Reprinted with permission from Wolters Kluwer Health and authors' permission, Naeser, Martin, Lundgren, et al., Improved Language in a Chronic Nonfluent Aphasia Patient After Treatment with CPAP and TMS, J Cogn and Beh Neurol, 2009, 23(1), 29-38. 


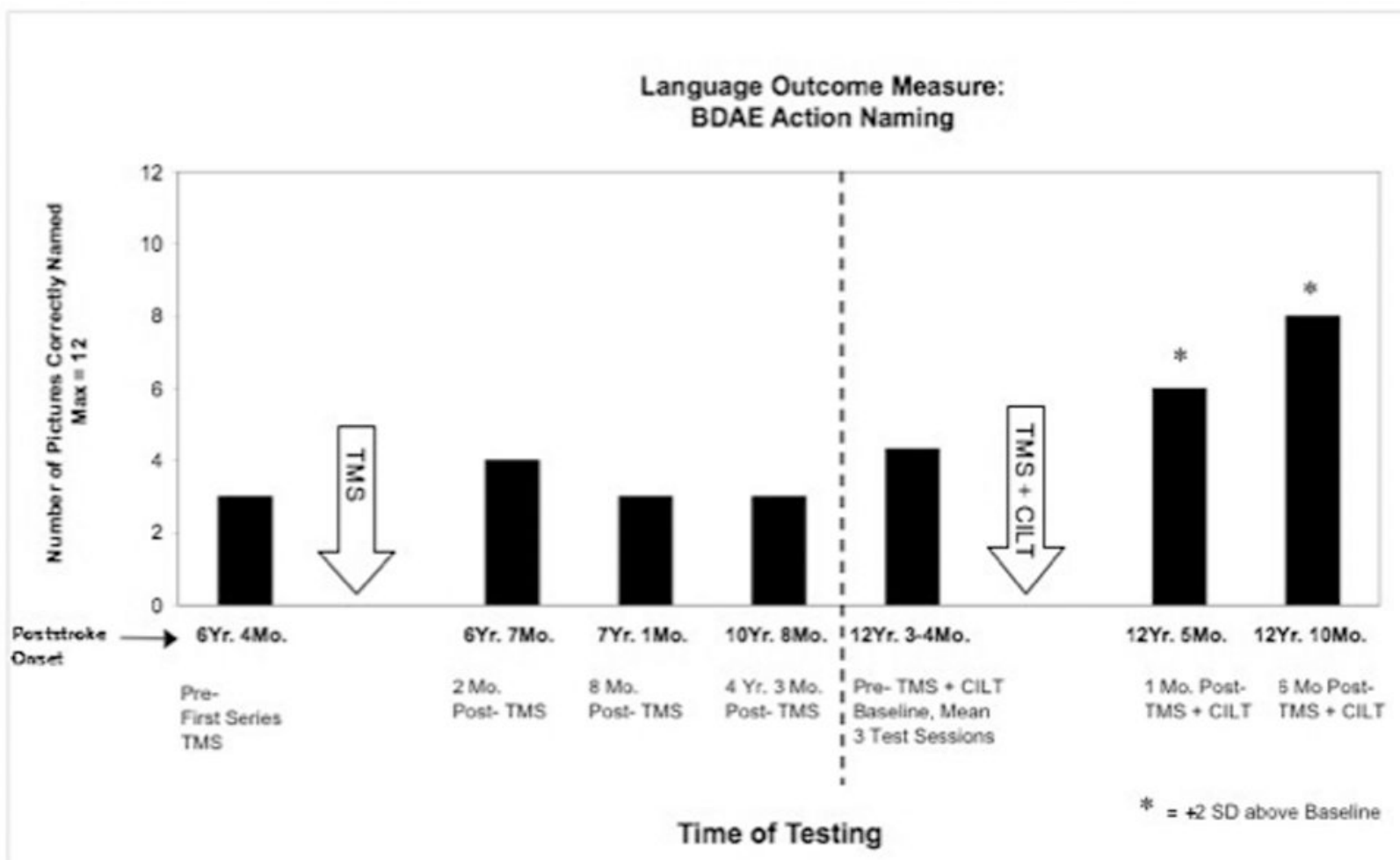

Figure 2.

Significant improvement in Action naming test scores post- Intervention (TMS+CILT) for a severe nonfluent, global aphasia patient. Bargraph shows significant improvement (>2 SD) in BDAE verb Action Naming at 1 and 6 mo. post- TMS+CILT, compared to baseline testing pre- Intervention. Bargraph also displays previous scores, pre- and post- TMS alone, without CILT. Abbreviations: TMS = transcranial magnetic stimulation; CILT = constraintinduced language therapy; BDAE = Boston Diagnostic Aphasia Exam. Reprinted from Restorative Neurology and Neuroscience, 28(4), Naeser, Martin, Treglia et al., Research with rTMS in the treatment of aphasia, 511-529, Copyright (2010), with permission from IOS Press. 

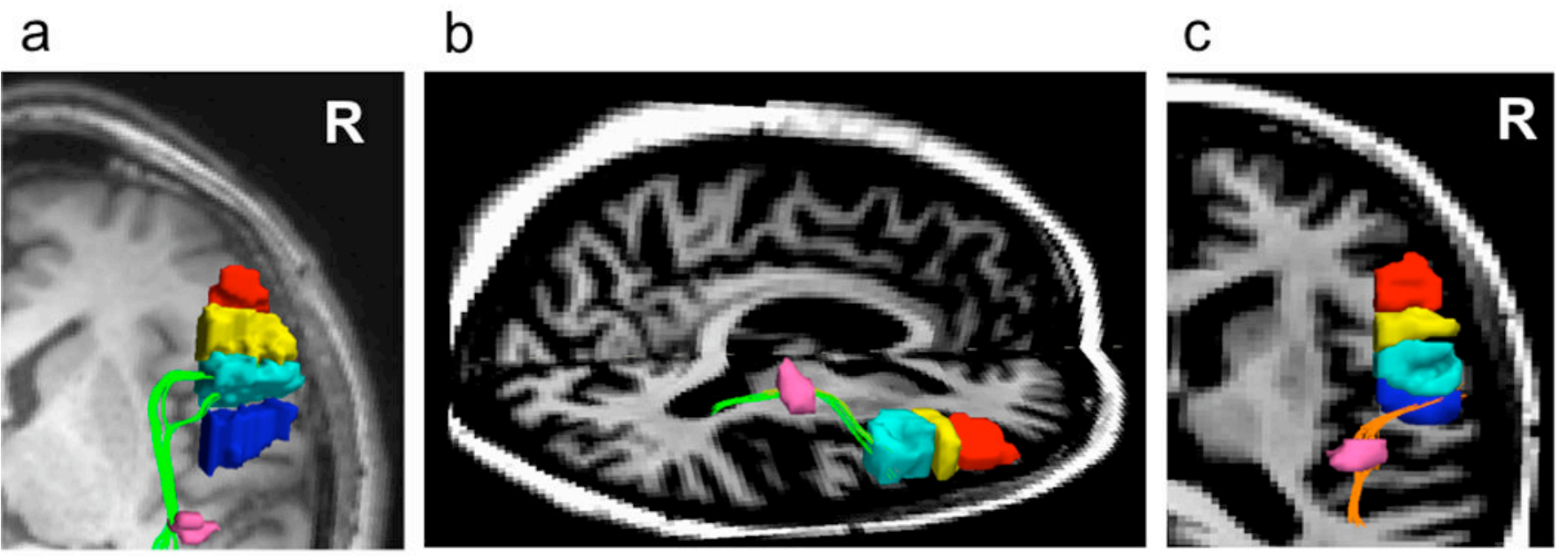

\section{Anterior Pars Triangularis Posterior Pars Triangularis Pars Opercularis}

\section{Ventral Premotor Cortex Arcuate Fasciculus Seed for Arcuate Fasciculus}

Figure 3.

$\mathrm{a}$ and $\mathrm{b}$ : White matter pathways from horizontal, mid-portion of arcuate fasciculus (AF, green) connect primarily with pars opercularis (light blue), not posterior pars triangularis (yellow) or anterior pars triangularis (red), in the right hemisphere; a: axial view, and b: sagittal view. c: White matter pathways from horizontal, mid-portion of AF (orange) also connect with ventral premotor cortex (dark blue) in the right hemisphere; c: axial view. The $\mathrm{R}$ pars opercularis and the $\mathrm{R}$ ventral premotor cortical areas are regions that may be important for recovery of speech in chronic stroke patients with nonfluent aphasia. Pink area represents seed point in the horizontal, mid-portion of the AF in these DTI studies with normal controls. 


\section{Table 1}

Language Testing for Mild Nonfluent Aphasia Patient, Pre- and Post- TMS36

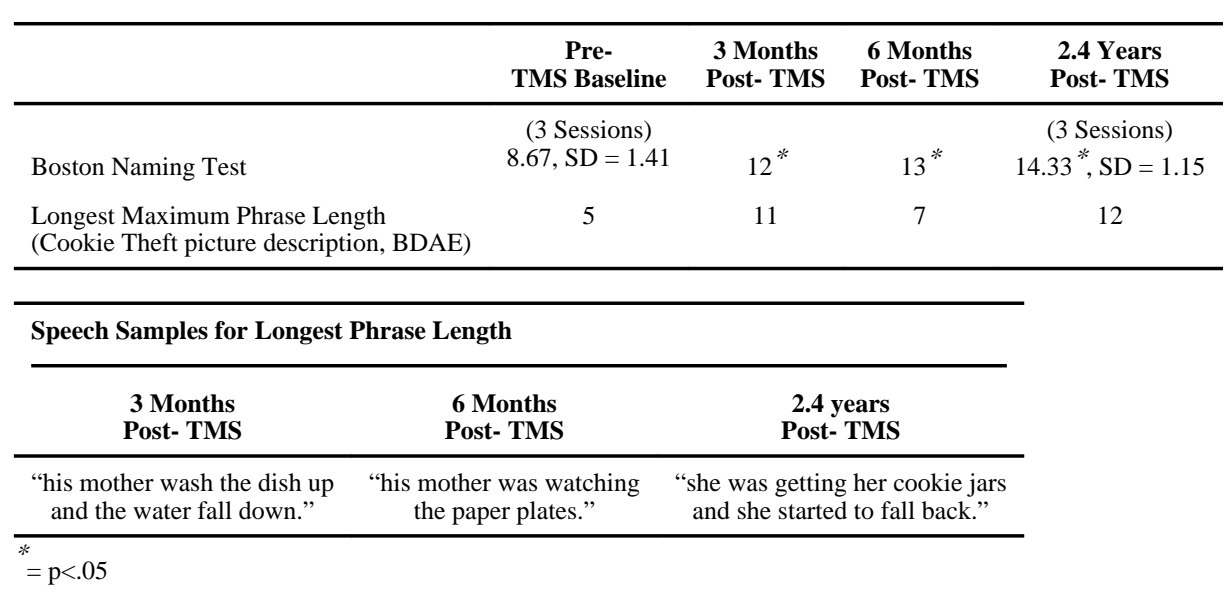




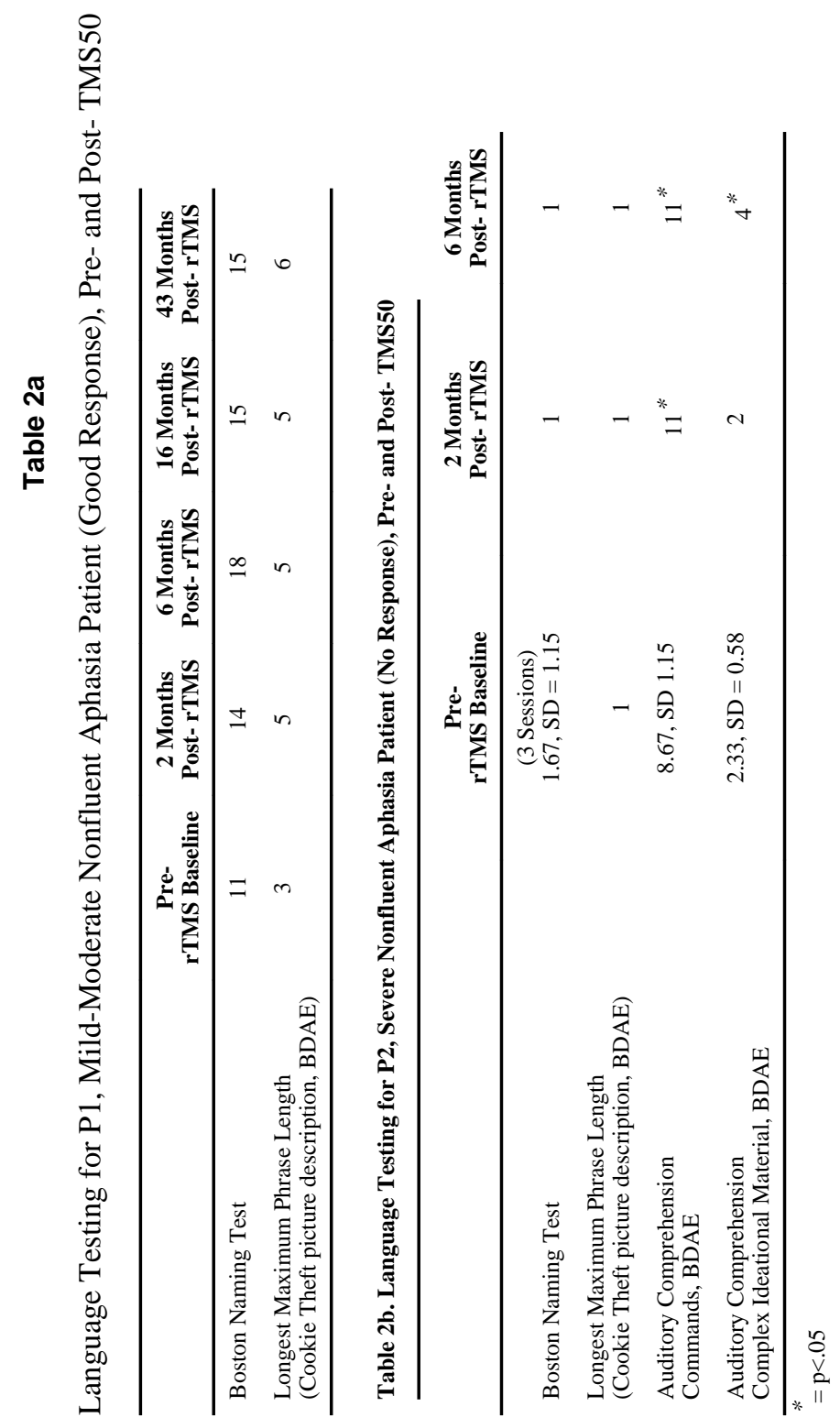

